\title{
New Ostrowski type inequalities for GA-convex functions
}

\author{
Hüsnü Anıl Çoban ${ }^{1 *}$, Imdat İscan ${ }^{2}$ Mehmet Kunt ${ }^{1}$ \\ ${ }^{1}$ Department of Mathematics, Faculty of Sciences, Karadeniz Technical University, 61080, Trabzon, Turkey \\ ${ }^{2}$ Department of Mathematics, Faculty of Sciences and Arts, Giresun University, 28200, Giresun, Turkey
}

Received: 18 August 2016, Accepted: 9 September 2016

Published online: 8 October 2016.

\begin{abstract}
In this work, we reveal a new equality for differentiable functions. By using this equality, we have some new Ostrowki type inequalities and some error estimates for the midpoint formula for functions whose derivatives in absolute values at certain powers are GA-convex.
\end{abstract}

Keywords: Ostrowski type inequalities, midpoint type inequalities, GA-convex function.

\section{Introduction}

The following result is known in the literature as Ostrowski's inequality [7];

Theorem 1. ([2,8]) Let $f:[a, b] \rightarrow \mathbb{R}$ be a differentiable mapping on $(a, b)$ with the property that $\left|f^{\prime}(t)\right| \leq M$ for all $t \in(a, b)$. Then

$$
\left|f(x)-\frac{1}{b-a} \int_{a}^{b} f(t) d t\right| \leq(b-a) M\left[\frac{1}{4}+\frac{\left(x-\frac{a+b}{2}\right)^{2}}{(b-a)^{2}}\right]
$$

for all $x \in[a, b]$.

The constant $\frac{1}{4}$ is the best possible it means that it cannot be replaced by a smaller constant. The inequality (1) can be expressed in the following form:

$$
\left|f(x)-\frac{1}{b-a} \int_{a}^{b} f(t) d t\right| \leq \frac{M}{b-a}\left[\frac{(x-a)^{2}+(b-x)^{2}}{2}\right] .
$$

For some results which generalize, improve and extend the inequalities (1) and (2) we refer the reader to the recent papers (see $[1,2,4,5,7,8,9])$.

In [6], Niculescu gave definition of GA-convex functions as follows:

Definition 1. A function $f: I \subset[0, \infty) \rightarrow \mathbb{R}$ is said to be GA-convex on $I$, if

$$
f\left(x^{t} y^{1-t}\right) \leq t f(x)+(1-t) f(y)
$$

holds for all $x, y \in I$ and $t \in[0,1]$.

In [3, Theorem 3.3], İşcan presented Hermite-Hadamard inequality for GA-s-convex functions in the second sense as follows: 
Theorem 2. ([3]) Suppose that $f: I \subset(0, \infty) \rightarrow \mathbb{R}$ is GA-s-convex function in the second sense and $a, b \in I$ with $a<b$. If $f \in L[a, b]$ then one has the inequalities:

$$
2^{s-1} f(\sqrt{a b}) \leq \frac{1}{\ln b-\ln a} \int_{a}^{b} \frac{f(x)}{x} d x \leq \frac{f(a)+f(b)}{s+1} .
$$

If one choose $s=1 \mathrm{in}(4)$, one has Hermite-Hadamard inequality for GA-convex functions as follows:

$$
f(\sqrt{a b}) \leq \frac{1}{\ln b-\ln a} \int_{a}^{b} \frac{f(x)}{x} d x \leq \frac{f(a)+f(b)}{2} .
$$

\section{New Ostorowski type inequalities}

We will use the following equality for proving our main results.

Lemma 1. Let $f: I \subset(0, \infty) \rightarrow \mathbb{R}$ be a differentiable function on $I^{\circ}$ such that $a, b \in I$ with $a<b$. If $f^{\prime} \in L[a, b]$ then

$$
f(x)-\frac{1}{\ln b-\ln a} \int_{a}^{b} \frac{f(u)}{u} d u=(\ln a-\ln b) \int_{0}^{1} p(t)\left(a^{t} b^{1-t}\right) f^{\prime}\left(a^{t} b^{1-t}\right) d t
$$

where

$$
p(t)=\left\{\begin{array}{cl}
t & , t \in\left[0, \frac{\ln b-\ln x}{\ln b-\ln a}\right] \\
t-1, & t \in\left(\frac{\ln b-\ln x}{\ln b-\ln a}, 1\right]
\end{array}\right.
$$

for all $x \in[a, b]$.

Proof. Using integration by parts then changing variables for following integral, we have

$$
\begin{aligned}
& (\ln a-\ln b) \int_{0}^{1} p(t)\left(a^{t} b^{1-t}\right) f^{\prime}\left(a^{t} b^{1-t}\right) d t \\
& =(\ln a-\ln b)\left[\int_{0}^{\frac{\ln b-\ln x}{\operatorname{nn} b-\ln a}} t\left(a^{t} b^{1-t}\right) f^{\prime}\left(a^{t} b^{1-t}\right) d t+\int_{\frac{\ln b-\ln x}{\ln b-\ln a}}^{1}(t-1)\left(a^{t} b^{1-t}\right) f^{\prime}\left(a^{t} b^{1-t}\right) d t\right] \\
& =(\ln a-\ln b)\left[\left.t \frac{f\left(a^{t} b^{1-t}\right)}{\ln a-\ln b}\right|_{0} ^{\frac{\ln b-\ln x}{\ln b-\ln a}}-\int_{0}^{\frac{\ln b-\ln x}{\ln b-\ln a}} \frac{f\left(a^{t} b^{1-t}\right)}{\ln a-\ln b} d t+\left.(t-1) \frac{f\left(a^{t} b^{1-t}\right)}{\ln a-\ln b}\right|_{\frac{\ln b-\ln x}{\ln b-\ln a}} ^{1}-\int_{\frac{\ln b-\ln x}{\ln b-\ln a}}^{1} \frac{f\left(a^{t} b^{1-t}\right)}{\ln a-\ln b} d t\right] \\
& =\left(\frac{\ln b-\ln x}{\ln b-\ln a}\right) f(x)-\frac{1}{\ln b-\ln a} \int_{x}^{b} \frac{f(u)}{u} d u+\left(1-\frac{\ln b-\ln x}{\ln b-\ln a}\right) f(x)-\frac{1}{\ln b-\ln a} \int_{a}^{u} d u \\
& =f(x)-\frac{1}{\ln b-\ln a} \int_{a}^{b} \frac{f(u)}{u} d u .
\end{aligned}
$$

This completes the proof.

Theorem 3. Let $f: I \subset(0, \infty) \rightarrow \mathbb{R}$ be a differentiable function on $I^{\circ}$ such that $a, b \in I$ with $a<b$ and $f^{\prime} \in L[a, b]$. If $\left|f^{\prime}\right|$ is GA-convex on $[a, b]$, then for all $x \in[a, b]$, we have

$$
\left|f(x)-\frac{1}{\ln b-\ln a} \int_{a}^{b} \frac{f(u)}{u} d u\right| \leq(\ln b-\ln a)\left[\left|f^{\prime}(a)\right|\left[\begin{array}{c}
H_{1}(a, b, x) \\
+H_{3}(a, b, x)
\end{array}\right]+\left|f^{\prime}(b)\right|\left[\begin{array}{c}
H_{2}(a, b, x) \\
+H_{4}(a, b, x)
\end{array}\right]\right]
$$


where

$$
\begin{aligned}
& H_{1}(a, b, x)=-\frac{1}{(\ln b-\ln a)^{3}}\left[(\ln b-\ln x)^{2} x+2(\ln b-\ln x+1) x-2 b\right], \\
& H_{2}(a, b, x)=\frac{1}{(\ln b-\ln a)^{3}}\left[\begin{array}{c}
(\ln b-\ln x)^{2} x+(\ln a-\ln b+2)(\ln b-\ln x) x \\
+(\ln a-\ln b+2) x+b \ln b-b \ln a-2 b
\end{array}\right], \\
& H_{3}(a, b, x)=\frac{1}{(\ln b-\ln a)^{3}}\left[\begin{array}{c}
-(\ln b-\ln x)^{2} x+(\ln b-\ln a-2)(\ln b-\ln x) x \\
+(\ln b-\ln a-2) x+a \ln b-a \ln a+2 a
\end{array}\right], \\
& H_{4}(a, b, x)=\frac{1}{(\ln b-\ln a)^{3}}\left[(\ln a-\ln x)^{2} x-2 a+2(\ln a-\ln x+1) x\right] .
\end{aligned}
$$

Proof. By using GA-convexity of $\left|f^{\prime}\right|$ on Lemma 1, we have

$$
\begin{aligned}
& \left|f(x)-\frac{1}{\ln b-\ln a} \int_{a}^{b} \frac{f(u)}{u} d u\right| \\
& \leq(\ln b-\ln a)\left[\int_{0}^{\frac{\ln b-\ln x}{\ln b-\ln a}} t\left(a^{t} b^{1-t}\right)\left|f^{\prime}\left(a^{t} b^{1-t}\right)\right| d t+\int_{\frac{\ln b-\ln x}{\ln b-\ln a}}^{1}(1-t)\left(a^{t} b^{1-t}\right)\left|f^{\prime}\left(a^{t} b^{1-t}\right)\right| d t\right] \\
& \leq(\ln b-\ln a)\left[\begin{array}{c}
\int_{0}^{\frac{\ln b-\ln x}{\ln m-\ln a}} t\left(a^{t} b^{1-t}\right)\left[t\left|f^{\prime}(a)\right|+(1-t)\left|f^{\prime}(b)\right|\right] d t \\
+\int_{\frac{\ln b-\ln x}{\ln b-\ln a}}^{1}(1-t)\left(a^{t} b^{1-t}\right)\left[t\left|f^{\prime}(a)\right|+(1-t)\left|f^{\prime}(b)\right|\right] d t
\end{array}\right] \\
& \leq(\ln b-\ln a)\left[\begin{array}{c}
\left|f^{\prime}(a)\right| \int_{0}^{\frac{\ln b-\ln x}{\ln b-\ln a}} t^{2}\left(a^{t} b^{1-t}\right) d t+\left|f^{\prime}(b)\right| \int_{0}^{\frac{\ln b-\ln x}{\ln b-\ln a}}\left(t-t^{2}\right)\left(a^{t} b^{1-t}\right) d t \\
+\left|f^{\prime}(a)\right| \int_{\frac{\ln b-\ln x}{\ln b-\ln a}}^{1}\left(t-t^{2}\right)\left(a^{t} b^{1-t}\right) d t+\left|f^{\prime}(b)\right| \int_{\frac{\ln b-\ln x}{\ln b-\ln a}}^{1}(1-t)^{2}\left(a^{t} b^{1-t}\right) d t
\end{array}\right] .
\end{aligned}
$$

Calculating appearing integrals, we have

$$
\begin{aligned}
& \int_{0}^{\frac{\ln b-\ln x}{\ln b-\ln a}} t^{2}\left(a^{t} b^{1-t}\right) d t=b \int_{0}^{\frac{\ln b-\ln x}{\ln b-\ln a}} t^{2}\left(\frac{a}{b}\right)^{t} d t \\
& =-\frac{1}{(\ln b-\ln a)^{3}}\left[(\ln b-\ln x)^{2} x+2(\ln b-\ln x+1) x-2 b\right]=H_{1}(a, b, x), \\
& \int_{0}^{\frac{\ln b-\ln x}{\operatorname{nn} b-\ln a}}\left(t-t^{2}\right)\left(a^{t} b^{1-t}\right) d t=b \int_{0}^{\frac{\ln b-\ln x}{\ln b-\ln a}}\left(t-t^{2}\right)\left(\frac{a}{b}\right)^{t} d t \\
& =\frac{1}{(\ln b-\ln a)^{3}}\left[\begin{array}{c}
(\ln b-\ln x)^{2} x+(\ln a-\ln b+2)(\ln b-\ln x) x \\
+(\ln a-\ln b+2) x+b \ln b-b \ln a-2 b
\end{array}\right]=H_{2}(a, b, x), \\
& \int_{\frac{\ln b-\ln x}{\ln b-\ln a}}^{1}\left(t-t^{2}\right)\left(a^{t} b^{1-t}\right) d t=b \int_{\frac{\ln b-\ln x}{\ln b-\ln a}}^{1}\left(t-t^{2}\right)\left(\frac{a}{b}\right)^{t} d t \\
& =\frac{1}{(\ln b-\ln a)^{3}}\left[\begin{array}{c}
-(\ln b-\ln x)^{2} x+(\ln b-\ln a-2)(\ln b-\ln x) x \\
+(\ln b-\ln a-2) x+a \ln b-a \ln a+2 a
\end{array}\right]=H_{3}(a, b, x), \\
& \int_{\frac{\ln b-\ln x}{\ln b-\ln a}}^{1}(1-t)^{2}\left(a^{t} b^{1-t}\right) d t=b \int_{\frac{\ln b-\ln x}{\ln b-\ln a}}^{1}(1-t)^{2}\left(\frac{a}{b}\right)^{t} d t \\
& =\frac{1}{(\ln b-\ln a)^{3}}\left[(\ln a-\ln x)^{2} x-2 a+2(\ln a-\ln x+1) x\right]=H_{4}(a, b, x) .
\end{aligned}
$$

(7) is obtained from the combination of (8)-(12). This completes the proof.

Corollary 1. In addition to the conditions of the Theorem 3, if we choose: 
(1) $\left|f^{\prime}(x)\right| \leq M$, for all $x \in[a, b]$, we have the following Ostrowski's type inequality for GA-convex functions

$$
\left|f(x)-\frac{1}{\ln b-\ln a} \int_{a}^{b} \frac{f(u)}{u} d u\right| \leq(\ln b-\ln a) M\left[H_{1}(a, b, x)+H_{3}(a, b, x)+H_{2}(a, b, x)+H_{4}(a, b, x)\right],
$$

(2) $x=\sqrt{a b}$, we have the following midpoint type inequality for GA-convex functions

$$
\begin{aligned}
\left|f(\sqrt{a b})-\frac{1}{\ln b-\ln a} \int_{a}^{b} \frac{f(u)}{u} d u\right| \leq & (\ln b-\ln a)\left[\left|f^{\prime}(a)\right|\left(H_{1}(a, b, \sqrt{a b})+H_{3}(a, b, \sqrt{a b})\right)\right. \\
& \left.+\left|f^{\prime}(b)\right|\left(H_{2}(a, b, \sqrt{a b})+H_{4}(a, b, \sqrt{a b})\right)\right]
\end{aligned}
$$

Theorem 4. Let $f: I \subset(0, \infty) \rightarrow \mathbb{R}$ be a differentiable function on $I^{\circ}$ such that $a, b \in I$ with $a<b$ and $f^{\prime} \in L[a, b]$. If $\left|f^{\prime}\right|^{q}$ is GA-convex on $[a, b]$ for $q \geq 1$, then for all $x \in[a, b]$, we have

$$
\begin{aligned}
\left|f(x)-\frac{1}{\ln b-\ln a} \int_{a}^{b} \frac{f(u)}{u} d u\right| & \leq(\ln b-\ln a)\left[\left(\frac{1}{2}\left(\frac{\ln b-\ln x}{\ln b-\ln a}\right)^{2}\right)^{1-\frac{1}{q}}\left(\begin{array}{c}
\left|f^{\prime}(a)\right|^{q} H_{5}(a, b, x) \\
+\left|f^{\prime}(b)\right|^{q} H_{6}(a, b, x)
\end{array}\right)^{\frac{1}{q}}\right. \\
& \left.+\left(\frac{1}{2}\left(\frac{\ln a-\ln x}{\ln b-\ln a}\right)^{2}\right)^{1-\frac{1}{q}}\left(\begin{array}{c}
\left|f^{\prime}(a)\right|^{q} H_{7}(a, b, x) \\
+\left|f^{\prime}(b)\right|^{q} H_{8}(a, b, x)
\end{array}\right)^{\frac{1}{q}}\right]
\end{aligned}
$$

where

$$
\begin{aligned}
& H_{5}(a, b, x)=\frac{-1}{q^{3}(\ln b-\ln a)^{3}}\left[q^{2}(\ln b-\ln x)^{2} x^{q}+2 q(\ln b-\ln x) x^{q}+2 x^{q}-2 b^{q}\right], \\
& H_{6}(a, b, x)=\frac{-1}{q^{3}(\ln b-\ln a)^{3}}\left[\begin{array}{c}
-q^{2}(\ln b-\ln x)^{2} x^{q}+q^{2}(\ln b-\ln a)(\ln b-\ln x) x^{q} \\
-2 q(\ln b-\ln x) x^{q}+q(\ln b-\ln a) x^{q}-2 x^{q}-q(\ln b-\ln a) b^{q}+2 b^{q}
\end{array}\right], \\
& H_{7}(a, b, x)=\frac{-1}{q^{3}(\ln b-\ln a)^{3}}\left[\begin{array}{c}
q^{2}(\ln b-\ln x)^{2} x^{q}-q^{2}(\ln b-\ln a)(\ln b-\ln x) x^{q} \\
+2 q(\ln b-\ln x) x^{q}-q(\ln b-\ln a) x^{q}+2 x^{q}-q(\ln b-\ln a) a^{q}-2 a^{q}
\end{array}\right], \\
& H_{8}(a, b, x)=\frac{1}{q^{3}(\ln b-\ln a)^{3}}\left[q^{2}(\ln a-\ln x)^{2} x^{q}+2 q(\ln a-\ln x) x^{q}+2 x^{q}-2 a^{q}\right] .
\end{aligned}
$$

Proof. By using Lemma 1, power mean inequality and GA-convexity of $\left|f^{\prime}\right|^{q}$, we have

$$
\begin{aligned}
& \left|f(x)-\frac{1}{\ln b-\ln a} \int_{a}^{b} \frac{f(u)}{u} d u\right| \\
& \leq(\ln b-\ln a)\left[\int_{0}^{\frac{\ln b-\ln x}{\ln b-\ln a}} t\left(a^{t} b^{1-t}\right)\left|f^{\prime}\left(a^{t} b^{1-t}\right)\right| d t+\int_{\frac{\ln b-\ln x}{\ln b-\ln a}}^{1}(1-t)\left(a^{t} b^{1-t}\right)\left|f^{\prime}\left(a^{t} b^{1-t}\right)\right| d t\right] \\
& \leq(\ln b-\ln a)\left[( \int _ { 0 } ^ { \frac { \operatorname { l n } b - \operatorname { l n } x } { \operatorname { l n } b - \operatorname { l n } a } } t d t ) ^ { 1 - \frac { 1 } { q } } \left(\left[\int_{0}^{\frac{\ln b-\ln x}{\ln b-\ln a}} t\left(a^{t} b^{1-t}\right)^{q}\left[t\left|f^{\prime}(a)\right|^{q}+(1-t)\left|f^{\prime}(b)\right|^{q}\right] d t\right)^{\frac{1}{q}}\right.\right. \\
& \left.+\left(\int_{\frac{\ln b-\ln x}{\ln b-\ln a}}^{1}(1-t) d t\right)^{1-\frac{1}{q}}\left(\int_{\frac{\ln b-\ln x}{\ln b-\ln a}}^{1}(1-t)\left(a^{t} b^{1-t}\right)^{q}\left[t\left|f^{\prime}(a)\right|^{q}+(1-t)\left|f^{\prime}(b)\right|^{q}\right] d t\right)^{\frac{1}{q}}\right] \\
& \leq(\ln b-\ln a)\left[\left(\frac{1}{2}\left(\frac{\ln b-\ln x}{\ln b-\ln a}\right)^{2}\right)^{1-\frac{1}{q}}\left(\left|f^{\prime}(a)\right|^{q} \int_{0}^{\frac{\ln b-\ln x}{\ln b-\ln a}} b^{q} t^{2}\left(\frac{a}{b}\right)^{q t} d t+\left|f^{\prime}(b)\right|^{q} \int_{0}^{\frac{\ln b-\ln x}{\ln b-\ln a}} b^{q} t(1-t)\left(\frac{a}{b}\right)^{q t} d t\right)^{\frac{1}{q}}\right.
\end{aligned}
$$




$$
\left.+\left(\frac{1}{2}\left(\frac{\ln a-\ln x}{\ln b-\ln a}\right)^{2}\right)^{1-\frac{1}{q}}\left(\left|f^{\prime}(a)\right|^{q} \int_{\frac{\ln b-\ln x}{\ln b-\ln a}}^{1} b^{q} t(1-t)\left(\frac{a}{b}\right)^{q t} d t+\left|f^{\prime}(b)\right|^{q} \int_{\frac{\ln b-\ln x}{\ln b-\ln a}}^{1} b^{q}(1-t)^{2}\left(\frac{a}{b}\right)^{q t} d t\right)^{\frac{1}{q}}\right]
$$

Calculating appearing integrals, we have

$$
\begin{aligned}
& \int_{0}^{\frac{\ln b-\ln x}{\ln b-\ln a}} b^{q} t^{2}\left(\frac{a}{b}\right)^{q t} d t \\
& =\frac{-1}{q^{3}(\ln b-\ln a)^{3}}\left[q^{2}(\ln b-\ln x)^{2} x^{q}+2 q(\ln b-\ln x) x^{q}+2 x^{q}-2 b^{q}\right]=H_{5}(a, b, x), \\
& \int_{0}^{\frac{\ln b-\ln x}{\ln b-\ln a}} b^{q} t(1-t)\left(\frac{a}{b}\right)^{q t} d t \\
& =\frac{-1}{q^{3}(\ln b-\ln a)^{3}}\left[\begin{array}{c}
-2 q(\ln b-\ln x) x^{q}+q(\ln b-\ln a) x^{q}-2 x^{q}-q(\ln b-\ln a) b^{q}+2 b^{q}
\end{array}\right]=H_{6}(a, b, x), \\
& \int_{\frac{\ln b-\ln x}{\ln b-\ln a}}^{1} b^{q} t(1-t)\left(\frac{a}{b}\right)^{q t} d t \quad \\
& =\frac{-1}{q^{3}(\ln b-\ln a)^{3}}\left[\begin{array}{c}
+2 q(\ln b-\ln x) x^{q}-q(\ln b-\ln a) x^{q}+2 x^{q}-q(\ln b-\ln a) a^{q}-2 a^{q}
\end{array}\right]=H_{7}(a, b, x), \\
& \int_{\frac{\ln b-\ln x}{\ln b-\ln a}}^{1} b^{q}(1-t)^{2}\left(\frac{a}{b}\right)^{q t} d t \\
& =\frac{1}{q^{3}(\ln b-\ln a)^{3}}\left[q^{2}(\ln a-\ln x)^{2} x^{q}+2 q(\ln a-\ln x) x^{q}+2 x^{q}-2 a^{q}\right]=H_{8}(a, b, x) .
\end{aligned}
$$

(15) is obtained from the combination of (16)-(20). This completes the proof.

Corollary 2. In addition to the conditions of the Theorem 4, if we choose:

(1) $\left|f^{\prime}(x)\right| \leq M$, for all $x \in[a, b]$, we have the following Ostrowski's type inequality for GA-convex functions

$$
\begin{aligned}
\left|f(x)-\frac{1}{\ln b-\ln a} \int_{a}^{b} \frac{f(u)}{u} d u\right| \leq & (\ln b-\ln a) M\left[\left(\frac{1}{2}\left(\frac{\ln b-\ln x}{\ln b-\ln a}\right)^{2}\right)^{1-\frac{1}{q}}\left(H_{5}(a, b, x)+H_{6}(a, b, x)\right)^{\frac{1}{q}}\right. \\
& \left.+\left(\frac{1}{2}\left(\frac{\ln a-\ln x}{\ln b-\ln a}\right)^{2}\right)^{1-\frac{1}{q}}\left(H_{7}(a, b, x)+H_{8}(a, b, x)\right)^{\frac{1}{q}}\right],
\end{aligned}
$$

(2) $x=\sqrt{a b}$, we have the following midpoint type inequality for GA-convex functions

$$
\begin{aligned}
& \left|f(\sqrt{a b})-\frac{1}{\ln b-\ln a} \int_{a}^{b} \frac{f(u)}{u} d u\right| \\
& \leq(\ln b-\ln a)\left(\frac{1}{8}\right)^{1-\frac{1}{q}}\left[\left(\begin{array}{c}
\left|f^{\prime}(a)\right|^{q} H_{5}(a, b, \sqrt{a b}) \\
+\left|f^{\prime}(b)\right|^{q} H_{6}(a, b, \sqrt{a b})
\end{array}\right)^{\frac{1}{q}}+\left(\begin{array}{c}
\left|f^{\prime}(a)\right|^{q} H_{7}(a, b, \sqrt{a b}) \\
+\left|f^{\prime}(b)\right|^{q} H_{8}(a, b, \sqrt{a b})
\end{array}\right)^{\frac{1}{q}}\right] .
\end{aligned}
$$


Theorem 5. Let $f: I \subset(0, \infty) \rightarrow \mathbb{R}$ be a differentiable function on $I^{\circ}$ such that $a, b \in I$ with $a<b$ and $f^{\prime} \in L[a, b]$. If $\left|f^{\prime}\right|^{q}$ is GA-convex on $[a, b]$ for $q \geq 1$, then for all $x \in[a, b]$, we have

$$
\begin{aligned}
\left|f(x)-\frac{1}{\ln b-\ln a} \int_{a}^{b} \frac{f(u)}{u} d u\right| & \leq(\ln b-\ln a)\left[\left(H_{9}(a, b, x)\right)^{1-\frac{1}{q}}\left(\left|f^{\prime}(a)\right|^{q} H_{1}(a, b, x)+\left|f^{\prime}(b)\right|^{q} H_{2}(a, b, x)\right)^{\frac{1}{q}}\right. \\
& \left.+\left(H_{10}(a, b, x)\right)^{1-\frac{1}{q}}\left(\left|f^{\prime}(a)\right|^{q} H_{3}(a, b, x)+\left|f^{\prime}(b)\right|^{q} H_{4}(a, b, x)\right)^{\frac{1}{q}}\right]
\end{aligned}
$$

where $H_{1}(a, b, x)-H_{4}(a, b, x)$ are defined as in Theorem 3 and

$$
\begin{aligned}
& H_{9}(a, b, x)=\frac{-1}{(\ln b-\ln a)^{2}}[(\ln b-\ln x+1) x-b] \\
& H_{10}(a, b, x)=\frac{1}{(\ln b-\ln a)^{2}}[(\ln x-\ln a-1) x+a] .
\end{aligned}
$$

Proof. By using Lemma 1, power mean inequality and GA-convexity of $\left|f^{\prime}\right|^{q}$, we have

$$
\begin{aligned}
& \left|f(x)-\frac{1}{\ln b-\ln a} \int_{a}^{b} \frac{f(u)}{u} d u\right| \\
& \leq(\ln b-\ln a)\left[\int_{0}^{\frac{\ln b-\ln x}{\ln b-\ln a}} t\left(a^{t} b^{1-t}\right)\left|f^{\prime}\left(a^{t} b^{1-t}\right)\right| d t+\int_{\frac{\ln b-\ln x}{\ln b-\ln a}}^{1}(1-t)\left(a^{t} b^{1-t}\right)\left|f^{\prime}\left(a^{t} b^{1-t}\right)\right| d t\right] \\
& \leq(\ln b-\ln a)\left[\left(\int_{0}^{\frac{\ln b-\ln x}{\ln b-\ln a}} t\left(a^{t} b^{1-t}\right) d t\right)^{1-\frac{1}{q}}\left(\int_{0}^{\frac{\ln b-\ln x}{\ln b-\ln a}} t\left(a^{t} b^{1-t}\right)\left[t\left|f^{\prime}(a)\right|^{q}+(1-t)\left|f^{\prime}(b)\right|^{q}\right] d t\right)^{\frac{1}{q}}\right. \\
& \left.+\left(\int_{\frac{\ln b-\ln x}{\ln b-\ln a}}^{1}(1-t)\left(a^{t} b^{1-t}\right) d t\right)^{1-\frac{1}{q}}\left(\int_{\frac{\ln b-\ln x}{\ln b-\ln a}}^{1}(1-t)\left(a^{t} b^{1-t}\right)\left[t\left|f^{\prime}(a)\right|^{q}+(1-t)\left|f^{\prime}(b)\right|^{q}\right] d t\right)^{\frac{1}{q}}\right] \\
& \leq(\ln b-\ln a)\left[\left(\int_{0}^{\frac{\ln b-\ln x}{\ln b-\ln a}} t\left(a^{t} b^{1-t}\right) d t\right)^{1-\frac{1}{q}}\left(\left|f^{\prime}(a)\right|^{q} \int_{0}^{\frac{\ln b-\ln x}{\ln b-\ln a}} b t^{2}\left(\frac{a}{b}\right)^{t} d t+\left|f^{\prime}(b)\right|^{q} \int_{0}^{\frac{\ln b-\ln x}{\ln b-\ln a}} b t(1-t)\left(\frac{a}{b}\right)^{t} d t\right)^{\frac{1}{q}}\right. \\
& \left.+\left(\int_{\frac{\ln b-\ln x}{\ln b-\ln a}}^{1}(1-t)\left(a^{t} b^{1-t}\right) d t\right)^{1-\frac{1}{q}}\left(\left|f^{\prime}(a)\right|^{q} \int_{\frac{\ln b-\ln x}{\ln b-\ln a}}^{1} b t(1-t)\left(\frac{a}{b}\right)^{t} d t+\left|f^{\prime}(b)\right|^{q} \int_{\frac{\ln b-\ln x}{\ln b-\ln a}}^{1} b(1-t)^{2}\left(\frac{a}{b}\right)^{t} d t\right)^{\frac{1}{q}}\right]
\end{aligned}
$$

Calculating appearing integrals, we have

$$
\begin{aligned}
& \int_{0}^{\frac{\ln b-\ln x}{\ln b-\ln a}} t\left(a^{t} b^{1-t}\right) d t=\frac{-1}{(\ln b-\ln a)^{2}}[(\ln b-\ln x+1) x-b]=H_{9}(a, b, x), \\
& \int_{\frac{\ln b-\ln x}{\ln b-\ln a}}^{1}(1-t)\left(a^{t} b^{1-t}\right) d t=\frac{1}{(\ln b-\ln a)^{2}}[(\ln x-\ln a-1) x+a]=H_{10}(a, b, x) .
\end{aligned}
$$

(23) is obtained from the combination of (9)-(12) and (24)-(26). This completes the proof.

Corollary 3. In addition to the conditions of the Theorem 5, if we choose: 
(1) $\left|f^{\prime}(x)\right| \leq M$, for all $x \in[a, b]$, we have the following Ostrowski's type inequality for GA-convex functions

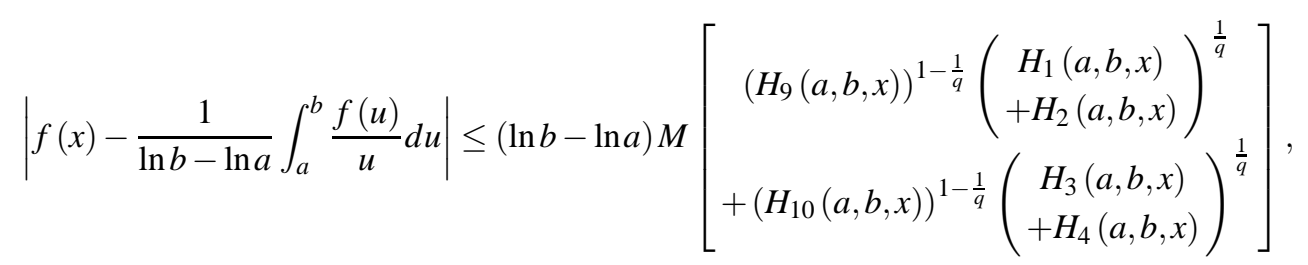

(2) $x=\sqrt{a b}$, we have the following midpoint type inequality for GA-convex functions

$$
\begin{aligned}
& \left|f(\sqrt{a b})-\frac{1}{\ln b-\ln a} \int_{a}^{b} \frac{f(u)}{u} d u\right| \\
& \leq(\ln b-\ln a)\left[\left(H_{9}(a, b, \sqrt{a b})\right)^{1-\frac{1}{q}}\left(\left|f^{\prime}(a)\right|^{q} H_{1}(a, b, \sqrt{a b})+\left|f^{\prime}(b)\right|^{q} H_{2}(a, b, \sqrt{a b})\right)^{\frac{1}{q}}\right. \\
& \left.+\left(H_{10}(a, b, \sqrt{a b})\right)^{1-\frac{1}{q}}\left(\left|f^{\prime}(a)\right|^{q} H_{3}(a, b, \sqrt{a b})+\left|f^{\prime}(b)\right|^{q} H_{4}(a, b, \sqrt{a b})\right)^{\frac{1}{q}}\right]
\end{aligned}
$$

Theorem 6. Let $f: I \subset(0, \infty) \rightarrow \mathbb{R}$ be a differentiable function on $I^{\circ}$ such that $a, b \in I$ with $a<b$ and $f^{\prime} \in L[a, b]$. If $\left|f^{\prime}\right|^{q}$ is GA-convex on $[a, b]$ for $q>1$ and $\frac{1}{p}+\frac{1}{q}=1$, then for all $x \in[a, b]$, we have

$$
\begin{aligned}
\left|f(x)-\frac{1}{\ln b-\ln a} \int_{a}^{b} \frac{f(u)}{u} d u\right| & \leq(\ln b-\ln a)\left[\left(\frac{1}{(p+1)}\left(\frac{\ln b-\ln x}{\ln b-\ln a}\right)^{p+1}\right)^{\frac{1}{p}}\left(\begin{array}{c}
\left|f^{\prime}(a)\right|^{q} H_{11}(a, b, x) \\
+\left|f^{\prime}(b)\right|^{q} H_{12}(a, b, x)
\end{array}\right)^{\frac{1}{q}}\right. \\
& +\left(\frac{1}{(p+1)}\left(\frac{\ln x-\ln a}{\ln b-\ln a}\right)^{p+1}\right)^{\frac{1}{p}}\left(\begin{array}{c}
\left|f^{\prime}(a)\right|^{q} H_{13}(a, b, x) \\
+\left|f^{\prime}(b)\right|^{q} H_{14}(a, b, x)
\end{array}\right)^{\frac{1}{q}}
\end{aligned}
$$

where

$$
\begin{aligned}
& H_{11}(a, b, x)=\frac{-1}{q^{2}(\ln b-\ln a)^{2}}\left[q(\ln b-\ln x) x^{q}+x^{q}-b^{q}\right] \\
& H_{12}(a, b, x)=\frac{1}{q^{2}(\ln b-\ln a)^{2}}\left[q(\ln b-\ln x) x^{q}-q(\ln b-\ln a) x^{q}+x^{q}+q(\ln b-\ln a) b^{q}-b^{q}\right] \\
& H_{13}(a, b, x)=\frac{1}{q^{2}(\ln b-\ln a)^{2}}\left[q(\ln b-\ln x) x^{q}+x^{q}-q(\ln b-\ln a) a^{q}-a^{q}\right] \\
& H_{14}(a, b, x)=\frac{1}{q^{2}(\ln b-\ln a)^{2}}\left[-q(\ln b-\ln x) x^{q}+q(\ln b-\ln a) x^{q}-x^{q}+a^{q}\right] .
\end{aligned}
$$

Proof. By using Lemma 1, Hölder inequality and GA-convexity of $\left|f^{\prime}\right|^{q}$, we have

$$
\begin{aligned}
& \left|f(x)-\frac{1}{\ln b-\ln a} \int_{a}^{b} \frac{f(u)}{u} d u\right| \\
& \leq(\ln b-\ln a)\left[\int_{0}^{\frac{\ln b-\ln x}{\ln b-\ln a}} t\left(a^{t} b^{1-t}\right)\left|f^{\prime}\left(a^{t} b^{1-t}\right)\right| d t+\int_{\frac{\ln b-\ln x}{\ln b-\ln a}}^{1}(1-t)\left(a^{t} b^{1-t}\right)\left|f^{\prime}\left(a^{t} b^{1-t}\right)\right| d t\right] \\
& \leq(\ln b-\ln a)\left[\left(\int_{0}^{\frac{\ln b-\ln x}{\ln b-\ln a}} t^{p} d t\right)^{\frac{1}{p}}\left(\int_{0}^{\frac{\ln b-\ln x}{\ln b-\ln a}}\left(a^{t} b^{1-t}\right)^{q}\left|f^{\prime}\left(a^{t} b^{1-t}\right)\right|^{q} d t\right)^{\frac{1}{q}}\right.
\end{aligned}
$$




$$
\begin{aligned}
& \left.+\left(\int_{\frac{\ln b-\ln x}{\ln b-\ln a}}^{1}(1-t)^{p} d t\right)^{\frac{1}{p}}\left(\int_{\frac{\ln b-\ln x}{\ln b-\ln a}}^{1}\left(a^{t} b^{1-t}\right)^{q}\left|f^{\prime}\left(a^{t} b^{1-t}\right)\right|^{q} d t\right)^{\frac{1}{q}}\right] \\
& \leq(\ln b-\ln a)\left[\left(\int_{0}^{\frac{\ln b-\ln x}{\ln b-\ln a}} t^{p} d t\right)^{\frac{1}{p}}\left(\int_{0}^{\frac{\ln b-\ln x}{\ln b-\ln a}}\left(a^{t} b^{1-t}\right)^{q}\left[t\left|f^{\prime}(a)\right|^{q}+(1-t)\left|f^{\prime}(b)\right|^{q}\right] d t\right)^{\frac{1}{q}}\right. \\
& \left.+\left(\int_{\frac{\ln b-\ln x}{\ln b-\ln a}}^{1}(1-t)^{p} d t\right)^{\frac{1}{p}}\left(\int_{\frac{\ln b-\ln x}{\ln b-\ln a}}^{1}\left(a^{t} b^{1-t}\right)^{q}\left[t\left|f^{\prime}(a)\right|^{q}+(1-t)\left|f^{\prime}(b)\right|^{q}\right] d t\right)^{\frac{1}{q}}\right] \\
& \leq(\ln b-\ln a)\left[\left(\frac{1}{(p+1)}\left(\frac{\ln b-\ln x}{\ln b-\ln a}\right)^{p+1}\right)^{\frac{1}{p}}\left(\begin{array}{c}
\left|f^{\prime}(a)\right|^{q} \int_{0}^{\frac{\ln b-\ln x}{\ln b-\ln a}} b^{q} t\left(\frac{a}{b}\right)^{q t} d t \\
+\left|f^{\prime}(b)\right|^{q} \int_{0}^{\ln b-\ln b-\ln a} b^{q}(1-t)\left(\frac{a}{b}\right)^{q t} d t
\end{array}\right)^{\frac{1}{q}}\right. \\
& \left.+\left(\frac{1}{(p+1)}\left(\frac{\ln x-\ln a}{\ln b-\ln a}\right)^{p+1}\right)^{\frac{1}{p}}\left(\begin{array}{c}
\left|f^{\prime}(a)\right|^{q} \int_{\frac{\ln b-\ln x}{\ln b-\ln a}}^{1} b^{q} t\left(\frac{a}{b}\right)^{q t} d t \\
+\left|f^{\prime}(b)\right|^{q} \int_{\frac{\ln b-\ln x}{\ln b-\ln a}}^{q} b^{q}(1-t)\left(\frac{a}{b}\right)^{q t} d t
\end{array}\right)^{\frac{1}{q}}\right] .
\end{aligned}
$$

Calculating appearing integrals, we have

$$
\begin{aligned}
\int_{0}^{\frac{\ln b-\ln x}{\ln b-\ln a}} b^{q} t\left(\frac{a}{b}\right)^{q t} d t & =\frac{-1}{q^{2}(\ln b-\ln a)^{2}}\left[q(\ln b-\ln x) x^{q}+x^{q}-b^{q}\right] \\
& =H_{11}(a, b, x), \\
\int_{0}^{\frac{\ln b-\ln x}{\ln b-\ln a}} b^{q}(1-t)\left(\frac{a}{b}\right)^{q t} d t & =\frac{1}{q^{2}(\ln b-\ln a)^{2}}\left[q(\ln b-\ln x) x^{q}-q(\ln b-\ln a) x^{q}+x^{q}+q(\ln b-\ln a) b^{q}-b^{q}\right] \\
& =H_{12}(a, b, x), \\
\int_{\frac{\ln b-\ln x}{\ln b-\ln a}}^{1} b^{q} t\left(\frac{a}{b}\right)^{q t} d t & =\frac{1}{q^{2}(\ln b-\ln a)^{2}}\left[q(\ln b-\ln x) x^{q}+x^{q}-q(\ln b-\ln a) a^{q}-a^{q}\right] \\
& =H_{13}(a, b, x), \\
\int_{\frac{\ln b-\ln x}{\ln b-\ln a}}^{1} b^{q}(1-t)\left(\frac{a}{b}\right)^{q t} d t & =\frac{1}{q^{2}(\ln b-\ln a)^{2}}\left[-q(\ln b-\ln x) x^{q}+q(\ln b-\ln a) x^{q}-x^{q}+a^{q}\right] \\
& =H_{14}(a, b, x) .
\end{aligned}
$$

(29) is obtained from the combination of (30)-(34). This completes the proof.

Corollary 4. In addition to the conditions of the Theorem 6, if we choose:

(1) $\left|f^{\prime}(x)\right| \leq M$, for all $x \in[a, b]$, we have the following Ostrowski's type inequality for GA-covex functions

$$
\begin{aligned}
\left|f(x)-\frac{1}{\ln b-\ln a} \int_{a}^{b} \frac{f(u)}{u} d u\right| & \leq(\ln b-\ln a) M\left[\left(\frac{1}{(p+1)}\left(\frac{\ln b-\ln x}{\ln b-\ln a}\right)^{p+1}\right)^{\frac{1}{p}}\left(\begin{array}{c}
H_{11}(a, b, x) \\
+H_{12}(a, b, x)
\end{array}\right)^{\frac{1}{q}}\right. \\
& \left.+\left(\frac{1}{(p+1)}\left(\frac{\ln x-\ln a}{\ln b-\ln a}\right)^{p+1}\right)^{\frac{1}{p}}\left(\begin{array}{c}
H_{13}(a, b, x) \\
+H_{14}(a, b, x)
\end{array}\right)^{\frac{1}{q}}\right]
\end{aligned}
$$


(2) $x=\sqrt{a b}$, we have the following midpoint type inequality for GA-convex functions

$$
\begin{aligned}
\left|f(\sqrt{a b})-\frac{1}{\ln b-\ln a} \int_{a}^{b} \frac{f(u)}{u} d u\right| & \leq(\ln b-\ln a)\left(\frac{1}{(p+1)}\left(\frac{1}{2}\right)^{p+1}\right)^{\frac{1}{p}}\left[\left(\begin{array}{c}
\left|f^{\prime}(a)\right|^{q} H_{11}(a, b, \sqrt{a b}) \\
+\left|f^{\prime}(b)\right|^{q} H_{12}(a, b, \sqrt{a b})
\end{array}\right)^{\frac{1}{q}}\right. \\
& +\left(\begin{array}{c}
\left|f^{\prime}(a)\right|^{q} H_{13}(a, b, \sqrt{a b}) \\
+\left|f^{\prime}(b)\right|^{q} H_{14}(a, b, \sqrt{a b})
\end{array}\right)
\end{aligned}
$$

Theorem 7. Let $f: I \subset(0, \infty) \rightarrow \mathbb{R}$ be a differentiable function on $I^{\circ}$ such that $a, b \in I$ with $a<b$ and $f^{\prime} \in L[a, b]$. If $\left|f^{\prime}\right|^{q}$ is GA-convex on $[a, b]$ for $q>1$ and $\frac{1}{p}+\frac{1}{q}=1$, then for all $x \in[a, b]$, we have

$$
\begin{aligned}
& \left|f(x)-\frac{1}{\ln b-\ln a} \int_{a}^{b} \frac{f(u)}{u} d u\right| \\
& \leq(\ln b-\ln a)\left[\left(H_{15}(a, b, x)\right)^{\frac{1}{p}}\left(\left|f^{\prime}(a)\right|^{q}\left(\frac{1}{2}\left(\frac{\ln b-\ln x}{\ln b-\ln a}\right)^{2}\right)+\left|f^{\prime}(b)\right|^{q}\left(\frac{1}{2}-\frac{1}{2}\left(\frac{\ln a-\ln x}{\ln b-\ln a}\right)^{2}\right)\right)^{\frac{1}{q}}\right. \\
& \left.+\left(H_{16}(a, b, x)\right)^{\frac{1}{p}}\left(\left|f^{\prime}(a)\right|^{q}\left(\frac{1}{2}-\frac{1}{2}\left(\frac{\ln b-\ln x}{\ln b-\ln a}\right)^{2}\right)+\left|f^{\prime}(b)\right|^{q}\left(\frac{1}{2}\left(\frac{\ln a-\ln x}{\ln b-\ln a}\right)^{2}\right)\right)^{\frac{1}{q}}\right]
\end{aligned}
$$

where

$$
H_{15}(a, b, x)=\int_{0}^{\frac{\ln b-\ln x}{\ln b-\ln a}} b^{p} t^{p}\left(\frac{a}{b}\right)^{p t} d t, \quad H_{16}(a, b, x)=\int_{\frac{\ln b-\ln x}{\operatorname{nn} b-\ln a}}^{1} b^{p}(1-t)^{p}\left(\frac{a}{b}\right)^{p t} d t .
$$

Proof. By using Lemma 1, Hölder inequality and GA-convexity of $\left|f^{\prime}\right|^{q}$, we have

$$
\begin{aligned}
& \left|f(x)-\frac{1}{\ln b-\ln a} \int_{a}^{b} \frac{f(u)}{u} d u\right| \\
& \leq(\ln b-\ln a)\left[\int_{0}^{\frac{\ln b-\ln x}{\operatorname{nn} b-\ln a}} t\left(a^{t} b^{1-t}\right)\left|f^{\prime}\left(a^{t} b^{1-t}\right)\right| d t+\int_{\frac{\ln b-\ln x}{\ln b-\ln a}}^{1}(1-t)\left(a^{t} b^{1-t}\right)\left|f^{\prime}\left(a^{t} b^{1-t}\right)\right| d t\right] \\
& \leq(\ln b-\ln a)\left[\left(\int_{0}^{\frac{\ln b-\ln x}{\ln b-\ln a}} t^{p}\left(a^{t} b^{1-t}\right)^{p} d t\right)^{\frac{1}{p}}\left(\int_{0}^{\frac{\ln b-\ln x}{\ln b-\ln a}}\left[t\left|f^{\prime}(a)\right|^{q}+(1-t)\left|f^{\prime}(b)\right|^{q}\right] d t\right)^{\frac{1}{q}}\right. \\
& \left.+\left(\int_{\frac{\ln b-\ln x}{\ln b-\ln a}}^{1}(1-t)^{p}\left(a^{t} b^{1-t}\right)^{p} d t\right)^{\frac{1}{p}}\left(\int_{\frac{\ln b-\ln x}{\ln b-\ln a}}^{1}\left[t\left|f^{\prime}(a)\right|^{q}+(1-t)\left|f^{\prime}(b)\right|^{q}\right] d t\right)^{\frac{1}{q}}\right] \\
& \leq(\ln b-\ln a)\left[\left(\int_{0}^{\frac{\ln b-\ln x}{\ln b-\ln a}} t^{p}\left(a^{t} b^{1-t}\right)^{p} d t\right)^{\frac{1}{p}}\left(\left|f^{\prime}(a)\right|^{q} \int_{0}^{\frac{\ln b-\ln x}{\ln b-\ln a}} t d t+\left|f^{\prime}(b)\right|^{q} \int_{0}^{\frac{\ln b-\ln x}{\operatorname{n} b-\ln a}}(1-t) d t\right)^{\frac{1}{q}}\right. \\
& \left.+\left(\int_{\frac{\ln b-\ln x}{\ln b-\ln a}}^{1}(1-t)^{p}\left(a^{t} b^{1-t}\right)^{p} d t\right)^{\frac{1}{p}}\left(\left|f^{\prime}(a)\right|^{q} \int_{\frac{\ln b-\ln x}{\ln b-\ln a}}^{1} t d t+\left|f^{\prime}(b)\right|^{q} \int_{\frac{\ln b-\ln x}{\ln b-\ln a}}^{1}(1-t) d t\right)^{\frac{1}{q}}\right] \\
& \leq(\ln b-\ln a)\left[\left(\int_{0}^{\frac{\ln b-\ln x}{\ln b-\ln a}} b^{p} t^{p}\left(\frac{a}{b}\right)^{p t} d t\right)^{\frac{1}{p}}\left(\left|f^{\prime}(a)\right|^{q}\left(\frac{1}{2}\left(\frac{\ln b-\ln x}{\ln b-\ln a}\right)^{2}\right)+\left|f^{\prime}(b)\right|^{q}\left(\frac{1}{2}-\frac{1}{2}\left(\frac{\ln a-\ln x}{\ln b-\ln a}\right)^{2}\right)\right)^{\frac{1}{q}}\right.
\end{aligned}
$$




$$
\left.+\left(\int_{\frac{\ln b-\ln x}{\ln b-\ln a}}^{1} b^{p}(1-t)^{p}\left(\frac{a}{b}\right)^{p t} d t\right)^{\frac{1}{p}}\left(\left|f^{\prime}(a)\right|^{q}\left(\frac{1}{2}-\frac{1}{2}\left(\frac{\ln b-\ln x}{\ln b-\ln a}\right)^{2}\right)+\left|f^{\prime}(b)\right|^{q}\left(\frac{1}{2}\left(\frac{\ln a-\ln x}{\ln b-\ln a}\right)^{2}\right)\right)^{\frac{1}{q}}\right] .
$$

Since the appearing integrals, we have

$$
\begin{gathered}
\int_{0}^{\frac{\ln b-\ln x}{\ln b-\ln a}} b^{p} t^{p}\left(\frac{a}{b}\right)^{p t} d t=H_{15}(a, b, x), \\
\int_{\frac{\ln b-\ln x}{\ln b-\ln a}}^{1} b^{p}(1-t)^{p}\left(\frac{a}{b}\right)^{p t} d t=H_{16}(a, b, x) .
\end{gathered}
$$

(37) is obtained from the combination of (38)-(40). This completes the proof.

Corollary 5. In addition to the conditions of the Theorem 7, if we choose:

(1) $\left|f^{\prime}(x)\right| \leq M$, for all $x \in[a, b]$, we have the following Ostrowski's type inequality for GA-convex functions

$$
\begin{aligned}
& \left|f(x)-\frac{1}{\ln b-\ln a} \int_{a}^{b} \frac{f(u)}{u} d u\right| \\
& \leq(\ln b-\ln a) M\left[\left(H_{15}(a, b, x)\right)^{\frac{1}{p}}\left(\left(\frac{1}{2}\left(\frac{\ln b-\ln x}{\ln b-\ln a}\right)^{2}\right)+\left(\frac{1}{2}-\frac{1}{2}\left(\frac{\ln a-\ln x}{\ln b-\ln a}\right)^{2}\right)\right)^{\frac{1}{q}}\right. \\
& \left.+\left(H_{16}(a, b, x)\right)^{\frac{1}{p}}\left(\left(\frac{1}{2}-\frac{1}{2}\left(\frac{\ln b-\ln x}{\ln b-\ln a}\right)^{2}\right)+\left(\frac{1}{2}\left(\frac{\ln a-\ln x}{\ln b-\ln a}\right)^{2}\right)\right)^{\frac{1}{q}}\right]
\end{aligned}
$$

(2) $x=\sqrt{a b}$, we have the following midpoint type inequality for GA-convex functions

$$
\left|f(\sqrt{a b})-\frac{1}{\ln b-\ln a} \int_{a}^{b} \frac{f(u)}{u} d u\right| \leq(\ln b-\ln a)\left[\begin{array}{c}
\left(H_{15}(a, b, \sqrt{a b})\right)^{\frac{1}{p}}\left(\frac{1}{8}\left|f^{\prime}(a)\right|^{q}+\frac{3}{8}\left|f^{\prime}(b)\right|^{q}\right)^{\frac{1}{q}} \\
+\left(H_{16}(a, b, \sqrt{a b})\right)^{\frac{1}{p}}\left(\frac{3}{8}\left|f^{\prime}(a)\right|^{q}+\frac{1}{8}\left|f^{\prime}(b)\right|^{q}\right)^{\frac{1}{q}}
\end{array}\right] .
$$

\section{Conclusions}

In this paper, we prove a new equality for differentiable functions. By using this equality, we have some new Ostrowki and midpoint type inequalities for functions whose derivatives in absolute values at certain powers are GA-convex.

\section{References}

[1] M. Alomari, M. Darus, Some Ostrowski’s type inequalities for convex functions with applications, RGMIA Res.Rep. Collect., 13(1) (2010), Article 3, 1-14.

[2] S. S. Dragomir, T. M. Rassias, Ostrowski type inequalities and applications in numerical integration, Kluwer Academic Publishers, 2002.

[3] İ. İşcan, Hermite-Hadamard type inequalities for GA-s-convex functions,Le Matematiche, Vol. LXIX (2014) 129-146.

[4] İ. İşcan, Ostrowski type inequalities for harmonically s-convex functions, Konuralp Jurnal of Mathematics, Volume 3, No 1 (2015) 63-74.

[5] İ. İşcan, S. Numan, Ostrowski type inequalities for harmonically quasi-convex functions, Elect. J. Math. Anal. App., 2(2) (2014) 189-198.

[6] C. P. Niculescu, Convexity according to the geometric mean, Math. Inequal. Appl. 3 (2) (2000), 155-167. 
[7] A. Ostrowski, Über die Absolutabweichung einer differentienbaren Funktionen von ihren Integralmittelwert. Comment. Math. Hel, 10 (1938), 226-227.

[8] M. E. Özdemir, Ç. Yıldız, New Ostrowski Type Inequalities for Geometrically Convex Functions, Int. J. Modern Math. Sci., 8(1) (2013) 27-35.

[9] E. Set, M. E. Özdemir, M. Z. Sarıkaya, New inequalities of Ostrowski's type for $s$-convex functions in the second sense with applications, Facta Uni. Ser. Math. Inform. 27(1) (2012) 67-82. 